\title{
Desenvolvimento de espécies florestais nativas em mata ciliar no Riacho Grilo, Sergipe
}

\author{
Dráuzio Correia Gama ${ }^{1 *}$ Robério Anastácio Ferreira ${ }^{2}$ Janisson Batista de Jesus ${ }^{3}$
}

\author{
$\mathbf{1}$ Mestre em Ciências Florestais, Brasil \\ ${ }^{2}$ Universidade Federal de Sergipe, Av. Marechal Rondon S/N, CEP 49100-000, São Cristóvão, SE, Brasil \\ 3 Universidade Federal do Rio Grande do Sul, campus Vale, Av. Bento Gonçalves, 9500, CEP 91501-970, Porto Alegre, RS, Brasil
}

\section{Artigo Original \\ *Autor correspondente:} drauziogama@hotmail.com

Palavras-chave:

Área degradada

Espécies florestais

Regeneração artificial

Restauração florestal

Keywords:

Degraded area

Forest species

Artificial regeneration

Forest restoration

Recebido em

2021/01/26

Aceito em

2021/10/21

Publicado em

2021/12/30

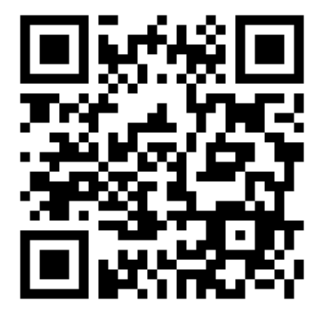

DOI:

https://doi.org/10.34062/afs. v8i4.11733

\section{(cc) BY}

RESUMO: O desmatamento das matas ciliares resulta em áreas degradadas que prejudicam a estabilidade dos ecossistemas associados a estes ambientes hídricos, necessitando, portanto, de estudos que indiquem espécies aptas para o uso em programas de recuperação das florestas ripárias. $\mathrm{O}$ estudo foi conduzido em um trecho de área perturbada de mata ciliar no Riacho Grilo, município de Salgado-SE, com o objetivo de testar espécies florestais nativas para recuperação de mata ciliar por meio de plantio de mudas. Utilizou-se de cinco espécies florestais: Anadenanthera macrocarpa (Benth) Brenan, Ceiba speciosa (A.St.-Hil.) Ravenna, Hymenaea stigonocarpa Mart. ex Hayne, Psidium guajava L. e Tabebuia aurea (Silva Manso) Benth. \& Hook.f. ex S.Moore com testes conduzidos por Delineamento em Blocos Casualizados (DBC) com quatro repetições e avaliadas em dois momentos: julho/2012 a maio/2013 como Avaliação I (AI) e setembro/2014 a maio/2015 como Avaliação II (AII). Computou-se mortalidade por contagem direta, e o desenvolvimento das mudas foi analisado pelo crescimento em altura do caule e diâmetro ao nível do solo (DNS), analisados pelo método Scott-Knott ao nível de $5 \%$ de significância. A taxa de mortalidade foi de 35,6\% (AI ) e 11,2\% (AII), com C. especiosa e T. aurea apresentando melhores médias em DNS nas duas avaliações (AI e AII). A. macrocarpa e C. especiosa seguida de $T$. aurea como melhores médias em altura em AI, e C. especiosa e A. macrocarpa em AII. Recomenda-se as espécies C. especiosa, A. macrocarpa e T. aurea para plantios de recuperação ambiental e/ou restauração florestal por meio do plantio de mudas na referida unidade de planejamento.

\section{Development of native forest species in riparian forest in Riacho Grilo, Sergipe}

ABSTRACT: The deforestation of riparian forests result in degraded areas that impair the stability of the ecosystems associated with these water environments, and therefore require studies that indicate species suitable for use in riparian forest recovery programs. The study was conducted in degraded riparian forest in the Grilo stream, in the country of Salgado-SE with the aim of testing tree species for riparian forest recovery through planting seedlings. Was used five forest species: Anadenanthera macrocarpa (Benth) Brenan, Ceiba speciosa (A.St.-Hil.) Ravenna, Hymenaea stigonocarpa Mart. ex Hayne, Psidium guajava L. e Tabebuia aurea (Silva Manso) Benth. \& Hook.f. ex S.Moore, with tests conducted by Casual Block Design (DBC) with four replications and evaluated in two moments: July/2012 to May/2013 (Evaluation 1 - E1) and September/2014 to May/2015 (Evaluation 2 E2). Was calculated the species mortality rate by direct counting, and the development of the seedlings was analyzed by the growth in height of shoot and diameter at ground level (DGL) and the averages analyzed by the Scott-Knott method in 5\% level of significance. The mortality rate was $35.6 \%$ (E1) and $11.2 \%$ (E2), with $C$. speciosa and T. aurea obtaining better DGL averages in the two evaluations (E1 and E2). A. macrocarpa and C. speciosa followed by T. aurea had better height averages in E1 and C. speciosa, and A. macrocarpa in E2. It is recommended species $C$. speciosa, A. macrocarpa and $T$. aurea in plantations for environmental restoration and/or forest restoration through planting seedlings in the planning unit. 
Gama et al.

Introdução

Os mananciais naturais constituem-se de fontes e reservatórios de água com atribuições de caráter ambiental, social e estratégico. Ambiental no que diz respeito aos processos ecológicos a eles associados, conectado, direta e indiretamente, à manutenção dos serviços ambientais; social quando relacionado ao desenvolvimento econômico da sociedade, pelo consumo, gerando emprego e receitas; e de caráter estratégico à segurança nacional, nos seus mais diversos aspectos.

A “Lei das Águas" (Lei no 9.433, de 08 de janeiro de 1997), que institui a Política Nacional dos Recursos Hídricos, ressalta a importância do consumo de água de mananciais naturais, principalmente rios, de forma responsável, respeitando o limite máximo permitido a garantir oferta constante desse recurso natural. Para tanto, as unidades de planejamento que respondem pela produção de água dependem da proteção e da conservação de suas características morfológicas e demais atributos, principalmente o seu componente vegetal (Ferreira et al. 2011, Silva et al. 2012).

Por sua vez, o "Código Florestal" (Lei no 12.651 de 25 de maio de 2012), realça entre outros termos que, a vegetação às margens de rios, comumente conhecida por mata ciliar, constitui-se, essencialmente, uma garantia necessária à sobrevida dos rios que a margeiam, seja protegendo-o e/ou auxiliando as recargas de água, além de abrigar a fauna local e promover o seu fluxo gênico. Observase, entretanto, que variavelmente às legislações ambientais têm sido descumpridas, resultando em diversas ações de degradação ambiental dessas vegetações ripárias, colocando em risco a produção e a qualidade das águas ou em determinadas situações, suprimindo sua fonte de forma irreversível (Varjabedian 2010, Oliveira e Engel 2017), por onde se faz necessário a restauração florestal desses ambientes.

Para Oliveira e Engel (2017), a restauração ecológica, com respeito à sua integridade, é uma atividade deliberada, que inicia ou acelera a recuperação de um ecossistema degradado, danificado, transformado ou totalmente destruído como resultado direto ou indireto das atividades humanas.

Dentre alguns métodos, tem-se o plantio misto que proporciona, com o rápido desenvolvimento das espécies, condição ideal do restabelecimento e funcionamento de processos ecossistêmicos, por exemplo, através de benefícios proporcionados com a oferta futura de recursos alimentares atrativos para a fauna e a estratificação de ambientes. Criando assim locais de refúgio de animais, locais para pouso de aves, bem como atraindo polinizadores e dispersores de sementes para a área (Oliveira et al. 2015). E favorecendo a sucessão e a regeneração natural em menor tempo e menor custo em todo o processo envolvido da restauração (Oliveira e Engel 2017).

As espécies florestais possuem ritmo de crescimento e desenvolvimento diferenciado (Silva et al. 2016), pelos quais são reconhecidos em grupos de sucessão ecológica. Em meio a eles, tem-se o grupo das espécies pioneiras e secundárias iniciais que são caracterizadas, dentre outras qualidades, segundo Budowski (1965), como heliófitas (embora suportando certo grau de sombreamento), de crescimento rápido e produtora de sementes pequenas a médias que podem ser dispersadas pelo vento ou por pássaros, além de longa viabilidade quando depositadas nos solos.

São grupos de espécies que contribuem para a restauração ecológico, criando com o seu sombreamento, microambientes favoráveis ao desenvolvimento das espécies de sucessão mais avançadas dependentes portanto dessas condições (Ignácio et al. 2007). As secundárias iniciais, por exemplo, são espécies arbóreas mais persistentes, pouca exigentes à fertilidade do solo, promovendo ampla distribuição, densidade e maior dominância, situando-se em um estágio mais avançado de sucessão quando comparado com as pioneira, contribuindo dessa forma com processos ecológicos mais acelerados na restauração ecológico (Budowski 1965, Ferretti et al. 1995, Paula et al. 2004, Lopes et al. 2011, Silva et al. 2016), o que tende a favorecer o avanço da sucessão dos grupos ecológicos mais rapidamente na restauração.

Por outro lado, ainda que se empreguem as técnicas corretas nos processos de recuperação e/ou restauração realizados por meio de regeneração artificial, com mudas de boa qualidade e plantios em período do ano ideal (com considerável taxa pluviométrica), têm sido recorrente altas taxas de mortalidade ou baixo estabelecimento das espécies; implicações com espécies invasoras e dificuldades do manejo do solo. Além de baixo incremento no crescimento das espécies, considerando-se o tempo esperado para a restauração (Almeida e Sanchez 2005, Galvão e Porfídio-da-Slva 2005, Schievenin et al. 2012, Santos et al. 2015).

Frente às essas questões críticas observadas historicamente, resultados não satisfatórios podem decorrer também pelo desconhecimento ecológico das espécies utilizadas, sendo-as diferentes da flora da unidade de planejamento ou sem histórico de sucessos em plantios nas mesmas condições ambientais e métodos empregados, uma vez que as chances de acertos são maiores com espécies arbóreas corretamente selecionadas (Brancalion et al. 2015). Sem ignorar, ainda, o monitoramento e as avaliações periódicas, os quais são indispensáveis a estimar a qualidade do desenvolvimento dos plantios e o nível de estabelecimento das espécies utilizadas (Rodrigues et al. 2009). 
Com isso, o presente estudo foi realizado com o objetivo de testar a sobrevivência e o desenvolvimento de espécies florestais de crescimento rápido para a recuperação de mata ciliar, por meio do plantio de mudas, no Riacho Grilo, Subbacia Hidrográfica do Rio Piauitinga, no estado de Sergipe.

\section{Material e Métodos}

Caracterização do local do estudo

O local de estudo compreende uma área de mata ciliar perturbada, com solo compactado, anteriormente empregada como pastagem, às margens do Riacho Grilo (10 $34^{\prime} 10^{\prime \prime}$ e $10^{\circ} 45^{\prime} 12^{\prime \prime} \mathrm{S}$ e $37^{\circ} 22^{\prime} 20^{\prime \prime}$ e $37^{\circ} 34^{\prime} 22^{\prime \prime} \mathrm{W}$ ) no município de Salgado-SE. A rede hidrográfica que abastece a região é a Sub-bacia do Rio Piauitinga, integrante da Bacia Hidrográfica do Rio Piauí pela sua margem esquerda (Figura1).

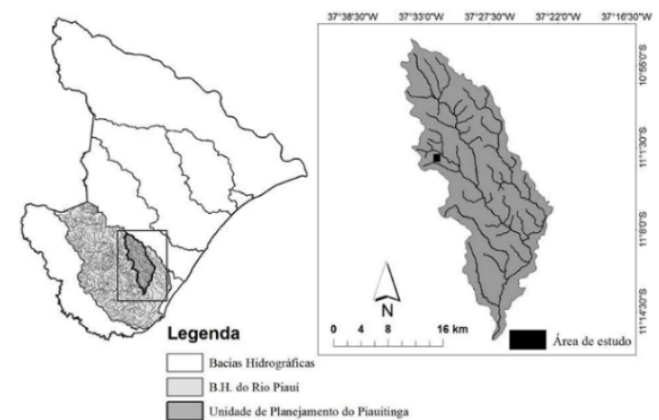

Figura 1. Localização da área de estudo de restauração de mata ciliar no Riacho Grilo, Subbacia Hidrográfica do rio Piauitinga, Sergipe.
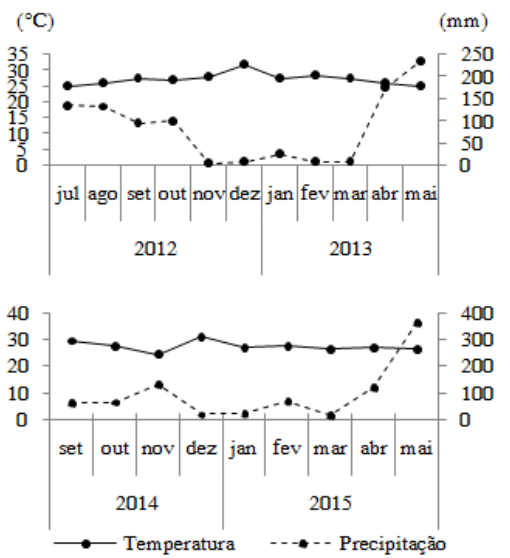

Figura 2. Precipitação acumulada e temperatura média adquiridos da Estação Meteorológica de Boquim-SE ao longo do período de estudo. Fonte: Sergipe (2015).

O município de Salgado tem precipitação e temperatura média anual, respectivamente, de 1.249 $\mathrm{mm}$ e $24,6^{\circ} \mathrm{C}$, com período chuvoso de março a julho em um clima do tipo C1A'a' (Megatérmico Subúmido) e C2A'a' (Megatérmico Subúmido Úmido) (Oliveira et al. 2012). Os tipos de solos encontrados são Argissolos Vermelho-Amarelo,
Latossolos Vermelho-Amarelo Distróficos e Neossolos Flúvico (Brasil 2014).

\section{Implantação do experimento}

$\mathrm{O}$ experimento foi implanto em julho de 2012. As mudas foram plantadas em Delineamento em Blocos Casualizados (DBC) com quatro repetições utilizando 180 indivíduos de cinco espécies florestais, duas pioneiras: Psidium guajava L. (goiabeira) e Tabebuia aurea (Silva Manso) Benth. \& Hook.f. ex S.Moore (craibeira). E três secundárias: Anadenanthera macrocarpa (Benth.) Brenan (angico), Ceiba speciosa (A.St.-Hil.) Ravenna (paineira) e Hymenaea stigonocarpa Mart. ex Hayne (jatobá-mirim).

Sendo-as espécies facilitadoras de rápido crescimento, fácil dispersão (paineira, angico), efeito paisagístico favorável (craibeira, jatobá-mirim) e atratividade da fauna (goiabeira) onde ainda, conforme Lorenzi (2008), possuem características ecológicas comuns, produzem anualmente grande quantidade de sementes viáveis e que são empregadas para reflorestamentos mistos em áreas perturbadas.

\section{Produção das mudas}

As espécies florestais utilizadas fazem parte da flora da própria unidade de planejamento, sendoas empregadas a manter o germoplasma da região. As suas mudas foram produzidas no Viveiro Florestal do Departamento de Ciências Florestais, da Universidade Federal de Sergipe em sacos plásticos pretos de polietileno $(18 \mathrm{~cm}$ x $25 \mathrm{~cm})$ com substrato a mistura de terra preta, areia lavada e esterco de curral nas proporções 3:1:1, respectivamente. Adicionando na composição os fertilizantes superfosfato simples $\left(1,5 \mathrm{~kg} / \mathrm{m}^{3}\right)$ e cloreto de potássio $\left(120 \mathrm{~g} / \mathrm{m}^{3}\right)$.

Após a emergência, cada plântula permaneceu recebendo adubação foliar em intervalos de 15 dias, sendo $50 \mathrm{~mL}$ da solução por planta, formado por cloreto de potássio $(60 \mathrm{~g})$ e sulfato de amônio ( $25 \mathrm{~g}$ ), diluídos em $10 \mathrm{~L}$ de água. Após serem retiradas do telado e permanecidas a pleno sol por um período de cinco meses, antes do plantio, a atingir rusticidade e qualidade morfofisiológica, as mudas mantiveram-se recebendo apenas as duas irrigações diárias (manhã e tarde) e apenas adubação foliar de cloreto de potássio realizado em intervalos de 15 dias.

\section{Realização do plantio}

Para a implantação, foram eliminadas as plantas invasoras encontradas no local através de roçada manual. A área não recebeu nenhum preparo do solo e foi cercada de arame farpado para evitar a entrada de animais presentes no entorno, o que também é uma medida para prevenção da área implantada e sua recuperação. O plantio foi realizado 
em covas $(30 \mathrm{~cm} \times 30 \mathrm{~cm} \times 30 \mathrm{~cm})$ e espaçamento de $1,5 \mathrm{~m} \times 1,5 \mathrm{~m}$.

Durante o plantio, realizou-se a adubação inicial de $200 \mathrm{~g}$ por muda de superfosfato simples e após 60 dias e adubação de cobertura com NPK (20:10:20) na quantidade de $150 \mathrm{~g}$ por muda dividida em duas partes distribuídas em duas covetas opostas à $15 \mathrm{~cm}$ da planta e $5 \mathrm{~cm}$ de profundidade, aproximadamente.

Também foram aplicadas iscas granuladas da marca comercial Mirex ${ }^{\circledR}$ para o controle de formigas. Para o controle de possíveis espécies invasoras, não foi necessário nenhum método, apenas coroamento em raio de $50 \mathrm{~cm}$ no entorno das mudas, pois as espécies encontravam-se isentas de qualquer sobreposição.

\section{Avaliação em campo e análise estatística}

O estabelecimento e o desenvolvimento das mudas utilizadas foram avaliados em dois períodos: Avaliação I (AI), a partir do plantio (julho/2012) e realizado ao longo de 300 dias (julho/2012 a maio/2013) e Avaliação II (AII), realizado ao longo de 240 dias (setembro/2014 a maio/2015), após 16 meses da última avaliação AI. Fez-se o monitoramento do índice de mortalidade e taxa de crescimentos primário e secundário, medindo o diâmetro ao nível do solo (DNS) com auxílio de paquímetro e a altura (da base à gema apical) utilizando-se de vara graduada.

A taxa de crescimento relativo (TCR\%) foi calculada através da equação TCR $(\%)=((\mathrm{Vf}-\mathrm{Vi}) /$ Vi), conforme Benincasa (1988), onde: $\mathrm{Vi}=$ Valor da variável no início do período considerado e $\mathrm{Vf}=$ Valor da variável no final do período considerado. Ao longo do monitoramento, avaliou-se o nível de mortalidade dos indivíduos por meio de contagem direta, considerando como plantas mortas os indivíduos ausentes na cova, com caule seco e sem folhas, como proposto por Brancalion et al. (2015).

Os dados foram submetidos à análise de variância (ANOVA) e, quando detectado diferença significativa, fez-se a comparação de médias por meio do método de Scott-Knott em nível de 5\% de significância, fazendo uso do software SISVAR ${ }^{\circledR}$ (Ferreira 2014)

\section{Resultados}

Ao final do estudo, computou-se uma mortalidade total de $42,7 \%$ das mudas utilizadas. Durante a Avaliação I (AI), realizada em maio de 2013 (após 300 dias), registrou-se uma mortalidade total de 64 indivíduos (35,6\% do total), com destaque para a espécie Psidium guajava com $92 \%$ de mortalidade, seguida por Hymenaea stigonocarpa (61\%) e Ceiba speciosa (22\%). Na Avaliação II (AII), realizada em maio de 2015 (após 240 dias), foi computado o total de 13 novas mortalidades $(11,2 \%$ dos 116 indivíduos remanescentes) com destaque para $P$. guajava (100\% de mortalidade) e $H$. stigonocarpa (28,6\%), conforme observado na Figura 3 .

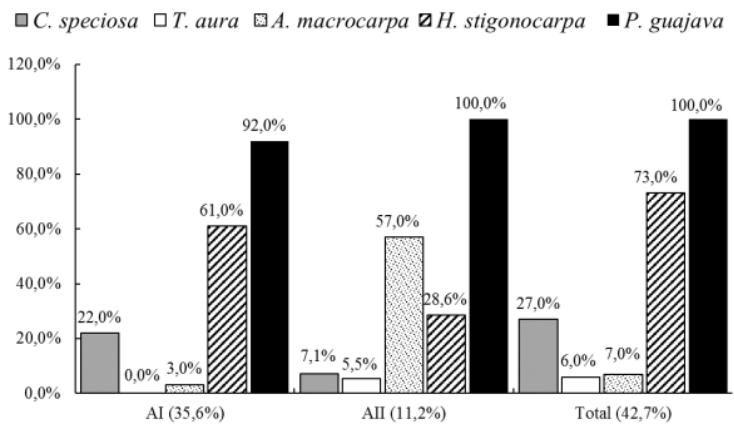

Figura 3. Taxa de mortalidade de espécies florestais referente às Avaliações I e II em área de estudo de restauração da mata ciliar no Riacho Grilo, Subbacia Hidrográfica do Rio Piauitinga, Sergipe.

Conforme se observa na Figura 4, referente a avaliação AI, ao longo do desenvolvimento ocorreram "picos" de crescimento das alturas de algumas espécies, representadas pelas retas dos gráficos. Dentre as espécies, $C$. speciosa apresentou destaque em acréscimo na altura, de forma abrupta dos 90 aos 120 dias de avaliação, mantendo-se constante em seguida. Nesse mesmo período ocorreu um decréscimo no incremento para a espécie $P$. guajava que se manteve constante até o final da avaliação.

Já Anadenanthera macrocarpa, a partir dos 30 dias de avaliação, despontou-se também com um decréscimo acentuado seguido de um crescimento, porém até os 270 dias de avaliação, onde voltou a crescer rapidamente. Quanto ao desenvolvimento das espécies Tabebuia aurea e H. stigonorcapa, estas mantiveram-se com baixos e constantes incrementos em suas alturas.

Sobre os crescimentos em diâmetros ao nível do solo (DNS), representado no gráfico pelas colunas, notam-se baixos incrementos das espécies durante todo período de avaliação, com uma ressalva para as espécies $T$. aurea e $C$. speciosa em que apresentaram consideráveis incrementos em diâmetro entre o início e os 120 dias de avaliação, mantendo-se constantes a partir dos 120 dias em diante e com A. macrocarpa com crescimentos constantes desprovidos de acréscimos abruptos.

Em AII (Figura 5), não se constatou variação abrupta no crescimento em altura, bem como também ao crescimento do diâmetro ao nível do solo ao contrário dos incrementos observados durante as avaliações em AI. 


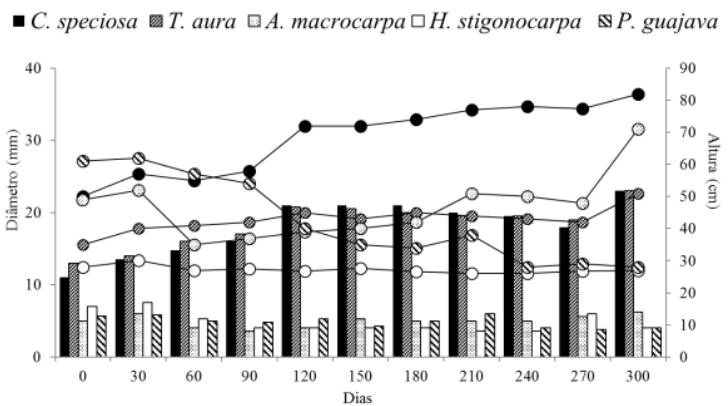

Figura 4. Incremento do crescimento em diâmetro ao nível do solo - DNS (eixo das colunas) e do crescimento em Altura (eixo das linhas) das mudas ao longo de 300 dias da Avaliação I das espécies florestais selecionadas para a área de estudo de restauração da mata ciliar no Riacho Grilo, Subbacia Hidrográfica do rio Piauitinga, Sergipe.

Apenas no crescimento em altura em AII foi notado algumas oscilações com baixos incrementos de acréscimos e decréscimos nada expressivos, tanto à espécie $C$. speciosa entre 90 e 180 dias como também em A. macrocarpa (120 e 150 dias) e $T$. aurea durante os 120 e 180 dias de observações.

Incrementos estáveis no crescimento em altura foram observados para a espécie $H$. stigonorcapa mantendo-se assim durante todo período de avaliação AI e AII.

Quanto a P. guajuva, embora tenha apresentado consideráveis incrementos durante as avaliações observadas em AI, a partir das primeiras avaliações em AII, ocorreu a mortalidade de seus indivíduos.

Para os crescimentos médios e relativos encontrados entre as espécies, por meio do método de Scott-Knott ao nível de 5\% de significância, após a análise de variância (ANOVA), detectou-se diferenças estatísticas entre os tratamentos com relação aos diâmetros ao nível do solo (Tabela 1) e as alturas dos indivíduos (Tabela 2).

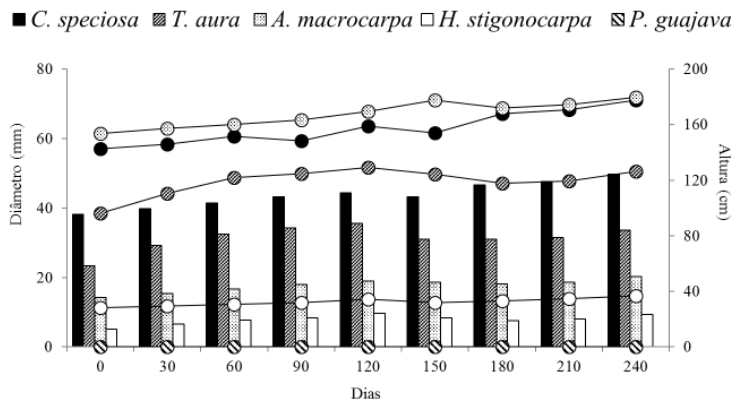

Figura 5. Incremento do crescimento em diâmetro ao nível do solo - DNS (eixo das colunas) e do crescimento em Altura (eixo das linhas) das mudas ao longo de 240 dias da Avaliação II das espécies florestais selecionadas para a área de estudo de restauração da mata ciliar no Riacho Grilo, Subbacia Hidrográfica do rio Piauitinga, Sergipe.
Tabela 1. Médias e taxa de crescimento relativo (TCR-\%) do diâmetro ao nível do solo (DNS) referente a Avaliação I e Avaliação II das espécies selecionadas à área de estudo de restauração da mata ciliar no Riacho Grilo, Sub-bacia Hidrográfica do Rio Piauitinga, Sergipe.

\begin{tabular}{|c|c|c|c|c|}
\hline \multirow{2}{*}{$\begin{array}{l}\text { Espécies } \\
\text { florestais }\end{array}$} & \multicolumn{2}{|c|}{$\begin{array}{l}\text { Avaliação I } \\
\text { (AI) }\end{array}$} & \multicolumn{2}{|c|}{$\begin{array}{c}\text { Avaliação II } \\
\text { (AII) }\end{array}$} \\
\hline & $\begin{array}{l}\text { DNS } \\
(\mathrm{mm})\end{array}$ & $\begin{array}{l}\text { TCR } \\
(\%)\end{array}$ & $\begin{array}{l}\text { DNS } \\
(\mathrm{mm})\end{array}$ & $\begin{array}{l}\text { TCR } \\
(\%)\end{array}$ \\
\hline C. speciosa & $20,87 \mathrm{a}$ & 106,53 & $50,11 \mathrm{a}$ & 30,50 \\
\hline T. aurea & $20,92 a$ & 74,24 & $33,64 b$ & 44,00 \\
\hline A. macrocarpa & $7,91 \mathrm{~b}$ & 92,66 & $20,17 \mathrm{c}$ & 43,31 \\
\hline H. stigononocarpa & $5,76 b$ & 64,91 & $4,52 \mathrm{~d}$ & 79,35 \\
\hline P. guajava & $4,52 \mathrm{~b}$ & 65,73 & $00,00 \mathrm{~d}$ & 00,00 \\
\hline
\end{tabular}

Tabela 2. Médias e taxa de crescimento relativo (TCR-\%) da altura $(\mathrm{H})$ referente a Avaliação I e Avaliação II das espécies selecionadas à área de estudo de restauração da mata ciliar no Riacho Grilo, Sub-bacia Hidrográfica do Rio Piauitinga, Sergipe.

\begin{tabular}{|c|c|c|c|c|}
\hline \multirow{2}{*}{$\begin{array}{l}\text { Espécies } \\
\text { florestais }\end{array}$} & \multicolumn{2}{|c|}{$\begin{array}{l}\text { Avaliação I } \\
\text { (AI) }\end{array}$} & \multicolumn{2}{|c|}{$\begin{array}{l}\text { Avaliação II } \\
\text { (AII) }\end{array}$} \\
\hline & $\begin{array}{c}\mathrm{H} \\
(\mathrm{cm})\end{array}$ & $\begin{array}{l}\text { TCR } \\
(\%)\end{array}$ & $\begin{array}{c}\mathrm{H} \\
(\mathrm{cm}) \\
\end{array}$ & $\begin{array}{l}\text { TCR } \\
(\%)\end{array}$ \\
\hline C. speciosa & $93,26 a$ & 100,30 & $181,78 \mathrm{a}$ & 24,50 \\
\hline T. aurea & $60,46 a$ & 85,19 & $127,47 b$ & 31,32 \\
\hline A. macrocarpa & $77,88 \mathrm{a}$ & 197,54 & $179,60 \mathrm{a}$ & 16,80 \\
\hline H. stigononocarpa & $25,63 b$ & 42,51 & $17,13 \mathrm{c}$ & 30,80 \\
\hline P. guajava & $28,00 \mathrm{~b}$ & 66,56 & $00,00 \mathrm{c}$ & 00,00 \\
\hline
\end{tabular}

As espécies C. speciosa e T. aurea, com ausência de diferenças estatísticas entre si, apresentaram médias superiores às demais no crescimento em DNS na Avaliação I, $20,87 \mathrm{~cm}$ e $20,92 \mathrm{~cm}$, respectivamente. No que se refere ao crescimento relativo do período de avaliação AI, $C$. speciosa se destacou por apresentar o maior crescimento (106,53\%), seguida por A. macrocarpa $(92,66 \%)$. Já no período de avaliação AII, todas tiveram diferenças significativas com $C$. speciosa apresentando maior média $(50,11 \mathrm{~cm})$ e $H$. stigononocarpa maior em crescimento relativo (79,35\%) seguida por T. aurea (44\%).

Quanto ao crescimento em altura, não há diferença estatística significativa entre as espécies com as maiores médias ( $C$. speciosa, T. aurea e $A$. macroparpa) em AI, tendo A. macrocarpa com maior crescimento relativo $(197,54 \%)$, seguida por C. speciosa $(100,30 \%)$. No segundo período de avaliação (AII), as três maiores médias foram observadas nas mesmas espécies da avaliação I (AI), porém havendo semelhança estatística apenas entre C. speciosa e A. macroparpa. Apesar disto, T. aurea 
apresentou maior TCR com 31,32\% seguida de $H$. stigononocarpa com 30,80\%.

\section{Discussões}

A mortalidade total de $42,7 \%$ entre todas as espécies estudadas é considerada alta, quando comparada ao mínimo exigido de até $10 \% \mathrm{em}$ projetos de recuperação ambiental, segundo Almeida e Sanchez (2005). Essa taxa tem como maior contribuição a mortalidade de $100 \%$ dos indivíduos de Psidium guajava, somado aos $61 \%$ de Hymenaea stigonocarpa e $22 \%$ de Ceiba speciosa.

Desse modo, o reflorestamento realizado foi compensado pelo desenvolvimento das espécies sobreviventes do local, com sobrevivência de $94 \%$ dos indivíduos de Tabebuia aurea e 93\% de Anadenanthera macrocarpa seguidas por $C$. speciosa $(73 \%)$.

A maior ocorrência de mortalidade das espécies aconteceu em AI, demonstrando dificuldades iniciais do estabelecimento. Com exceção de $T$. aurea com nenhuma mortalidade nesse período.

As condições edafoclimáticas do local, como solos compactados e precipitações irregulares, interferindo no desenvolvimento do sistema radicular e nas taxas fotossintéticas de algumas espécies, provavelmente tenham sido as principais causas de índices de mortalidade, principalmente durante o período inicial de estabelecimento (AI). Para Carnevali et al. (2016), as condições edáficas do solo degradado estão entre os fatores de maior impacto na sobrevivência das plantas.

Pires et al. (2012) ainda esclarecem que o estabelecimento inicial de espécies arbóreas é uma das fases mais críticas, por estarem vulneráveis as intempéries ambientais, além do comportamento aos níveis de luz, umidade e nutrientes do solo diferencialmente a determinadas espécies quanto ao crescimento e sobrevivência.

O arranjo espacial do plantio de mudas pode ter sido, possivelmente, outro fator de mortalidade. Bem como a presença de $P$. guajuva, uma vez que a espécie tem sido apontada como supridora da flora nativa nos estágios iniciais de sucessão pelo efeito alelopático no crescimento das outras espécies, segundo Chapla e Campos (2010).

Por outro lado, por mais que seja um resultado indesejado, a mortalidade das mudas constatada no presente estudo não é maior que a encontrada em outras áreas submetidas a restauração ecológica como pode se ver em Salviano et al. (2013) que, com apenas 180 dias de avaliação em GilbuésPI, observaram $35,4 \%$ de mortalidade e $33,3 \%$ foi encontrado em Santos et al. (2015) com 60 dias de avaliação em Cuiabá-MT.

Por iguais 540 dias de avaliação, Rodrigues et al. (2010) em Pontal do Paranapanema-SP, Silva et al. (2015) no sudoeste de Minas Gerais e Pereira et al. (2012) em Uberlândia-MG, obtiveram diferentes taxas de mortalidade, respectivamente, $50,2 \%, 22,9 \%$ e $63,64 \%$. Gama et al. (2020) notaram a mortalidade de $49 \%$ do total dos indivíduos entre as espécies estudadas aos 630 dias de avaliação em Lagarto-SE. Já Schievenin et al. (2012) em Sorocaba-SP, ao final de 720 dias, constataram a mortalidade de $31,3 \%$ dos indivíduos entre as espécies estudadas.

Quanto ao incremento no crescimento, nos primeiros períodos de avaliação (AI) do presente estudo, foi possível notar a espécie $C$. speciosa apresentando altos picos em altura, confirmando o seu estabelecimento sem maiores dificuldades.

Já durante a avaliação AII, encontrou-se pico máximo em A. macrocarpa e T. aurea, mantendo-se com crescimentos constantes ao longo do estudo. E picos mínimos e máximos variáveis em $C$. speciosa.

$\mathrm{O}$ crescimento em diâmetro ao nível do solo (DNS) bastante expressiva nas espécies $T$. aurea e $C$. speciosa e o crescimento em altura de forma significativo nas espécies $C$. speciosa, $T$. aurea e $A$. macrocarpa, denota o caráter ecológico peculiar de espécies de crescimento rápido a atingir a codominância do dossel das florestas, quando relacionada a estrutura vertical, expressando-se com isso a conduta dominante em ocupar uma área mais rapidamente, significativo para o exercício de sua função ecológica em favorecer as espécies de sucessão subsequentes.

Vale salientar que a estagnação do crescimento de algumas espécies, bem como o seu decréscimo abrupto em determinado período, a exemplo de $H$. stigonorcapa, deve-se a redução de indivíduos da área, provocada pela mortalidade, o que tende a influenciar no cômputo dos crescimentos de forma a diminuir os seus valores médios.

Por outro lado, nota-se que as espécies sobreviventes conseguiram incrementar mais biomassa em seus crescimentos durante o seu desenvolvimento em AII, superando, possivelmente, as dificuldades ambientais encontradas que retardaram o seu desenvolvimento no início.

Esse comportamento pode ser observado em alguns autores que apresentaram em seus estudos taxas de mortalidades consideráveis, a exemplo de Salviano et al. (2013), que ao final de 180 dias de avaliação, apresentaram incrementos em altura para as espécies sobreviventes Anadenanthera macrocarpa (Benth.) Brenan, Albizia lebbeck (L.) Benth. e Ochroma pyramidale (Cav. Ex Lam.), respectivamente, $114,45 \%, 110,14 \%$ e $109,83 \%$ de taxas de crescimento.

Da mesma forma, Gama et al. (2020), ao final de 630 dias de avaliação, encontraram taxa de crescimento em altura de $452,1 \%$ em Tapirira guianensis Aubl e 280,6\% em Guazuma ulmifolia Lam., entre as espécies estudadas.

Demonstrando, portanto, que algumas espécies conseguem se estabelecer e desenvolver-se, resistindo em algum momento as condições 
adversas, como observado com $C$. speciosa no presente estudo.

Ainda que se esperasse um contínuo ou maior desenvolvimento de todas as espécies no presente estudo, principalmente em crescimento em altura, por ser esse um parâmetro característico de grupo ecológico de espécies de crescimento rápido, autores como Santos et al. (2004) pontuam que o grupo ecológico de determinadas espécies nem sempre correspondem ao teórico desenvolvimento esperado, podendo indicar comportamentos opostos em diferentes áreas, demonstrando que as estratégias das espécies são, via de regra, conjuntos interativos complexos de adaptações cujas propriedades emergentes podem extrapolar simplificações.

\section{Conclusões}

Das espécies testadas, $C$. speciosa e $A$. macrocarpa em crescimento em altura e $C$. speciosa $e T$. áurea em crescimento em diâmetro, mostraramse prósperas e as melhores indicadas para plantios mistos dessa natureza na referida unidade de planejamento.

\section{Referências}

Almeida ROPO, Sánchez LE (2005) Revegetação de Áreas de Mineração: Critérios de Monitoramento e Avaliação do Desempenho. Revista Árvore, 29 (1): 47-54. DOI: http://dx.doi.org/10.1590/S010067622005000100006

Benincasa MMP (1988) Análise de crescimento de plantas (noções básicas). Jaboticabal: FCAVUNESP, $41 \mathrm{p}$.

Brancalion PHS, Viani RAG, Rodrigues RR, Gandolfi, S (2015) Avaliação e monitoramento de áreas em processo de restauração. In: Martins SV, Restauração ecológica de ecossistemas degradados. Viçosa: Editora UFV, $2^{\circ}$ ed. 293p.

Brasil (2014) Empresa Brasileira de Pesquisa Agropecuária, EMBRAPA. Sistema brasileiro de classificação de solos. Rio de Janeiro: Embrapa Solos, $3^{\circ}$ ed. 306p.

Budowski, A (1965) Distribution of tropical American rain forest species in the light of successional progresses. Turrialba, 15: 40-2.

Carnevali NHDS, Santiago EF, Daloso DDM, Carnevali, TDO, Oliveira MTD (2016) Sobrevivência e crescimento inicial de espécies arbóreas nativas implantadas em pastagem degradada. Floresta, $46 \quad$ (2): 277-286. DOI: http://dx.doi.org/10.5380/rf.v46i2.42881

Chapla TE, Campos, JB (2010) Allelopathic evidence in exotic guava (Psidium guajava L.). Brazilian archives of Biology and Technology,
53: 1359-1362. DOI:https://doi.org/10.1590/S1516$\underline{89132010000600012}$

Ferreira DF (2014) Sisvar: a Guide for its Bootstrap procedures in multiple comparisons. Ciência Agrotecnologia, 38 (2): 109-112. DOI: https://doi.org/10.1590/S1413-70542014000200001

Ferreira RA, Aguiar Neto ADO, Santos TIS, Santos, BL, Matos ELD (2011) Nascentes da Sub-bacia hidrográfica do rio Poxim, Estado de Sergipe: da degradação à restauração. Revista Árvore, 35 (2): 265-277. DOI: https://doi.org/10.1590/S0100$\underline{67622011000200011}$

Ferretti AR, Kageyama PY, Árboz GF, Santos JD, Barros MIA, Lorza RF, Oliveira C (1995) Classificação das espécies arbóreas em grupos ecológicos para revegetação com nativas no Estado de São Paulo. Florestar Estatístico, 3 (7): 73-77.

Galvão APM, Porfídio-da-Silva V (2005) Restauração Florestal: fundamentos e estudos de caso. Colombo: Embrapa Floresta, $1^{\circ}$ ed. 139p.

Gama DC, Ferreira RA, Jesus JBD (2020) Establishment of native forest species in riparian recovery forest in the Piauitinga River, Sergipe, Brazil. Floresta, $50 \quad$ (2): 1325-1334. DOI: http://dx.doi.org/10.5380/rf.v50i2.62987

Ignácio ED, Attanasio CM, Toniato MTZ (2007) Monitoramento de plantios de restauração de florestas ciliares: microbacia do Ribeirão São João, Mineiros do Tietê, SP. Revista Instituto Florestal, 19 (2): $137-148$

Lopes SDF, Schiavini I, Prado Júnior JAD, Gusson AE, Souza Neto, AR, Vale VSD, Dias Neto OC (2011) Caracterização ecológica e distribuição diamétrica da vegetação arbórea em um remanescente de floresta estacional semidecidual na fazenda experimental do Gloria, Uberlândia, MG. Bioscience Journal, 27 (2): 322-335.

Lorenzi H (2008) Árvores Brasileiras: manual de identificação de plantas arbóreas do Brasil. Nova Odessa: Instituto Plantarum, v. 1, $5^{\circ}$ ed. 384p.

Oliveira DG, Ferreira RA, Mello AAD, Oliveira RSCD, Oliveira RSCD (2012) Análise da vegetação em nascentes da Bacia Hidrográfica do Rio Piauitinga, Salgado, SE. Revista Árvore, 36 (1): $127-$ 141. DOI: https://doi.org/10.1590/S0100$\underline{67622012000100014}$

Oliveira MC, Passos FB, Ribeiro JF, Aquino FG, Oliveira FF, Sousa SR (2015) Crescimento de espécies nativas em um plantio de recuperação de Cerrado sentido restrito no Distrito Federal, 
Gama et al.

Brasil. Revista Brasileira de Biociências, 13 (1): 2532.

Oliveira RED, Engel VL (2017) A restauração florestal na Mata Atlântica: três décadas em revisão. Revista Ciência, Tecnologia \& Ambiente, 5 (1): 40-48. DOI: http://dx.doi.org/10.4322/23596643.05101

Paula AD, Silva AFD, Marco Júnior PD, Santos FAMD, Souza ALD (2004) Sucessão ecológica da vegetação arbórea em uma Floresta Estacional Semidecidual, Viçosa, MG, Brasil. Acta Botanica Brasilica, 18 (3): 407-423.

Pereira JS, Abreu CFNRD, Pereira Junior RA, Rodrigues SC (2012) Avaliação da taxa de sobrevivência e crescimento de espécies arbóreas utilizadas na recuperação de área degradada. Revista Geonorte, Edição especial, 1 (4): 138 -148.

Pires LA, Cardoso VJM, Rodrigues RR, Joly CA (2012) Sobrevivência e crescimento inicial de Ocoteapulchella (Lauraceae) em uma floresta de restinga da Ilha do Cardoso-SP. Rodriguésia, 63 (4): 763-774. DOI: https://doi.org/10.1590/S2175$\underline{78602012000400002}$

Rodrigues ER, Monteiro R, Cullen Junior (2010) Dinâmica inicial da composição florística de uma área restaurada na região do Pontal do Paranapanema, São Paulo, Brasil. Revista Árvore, 34 (5): 853-861. DOI: https://doi.org/10.1590/S0100$\underline{67622010000500010}$

Rodrigues RR, Lima RAF, Gandolfi S, Nave AG (2009) On the restoration of high diversity forests: 30 years of experience in the Brazilian Atlantic Forest. Biological Conservation, 142 (6): 12421251.

DOI:

https://doi.org/10.1016/j.biocon.2008.12.008

Salviano AAC, Lima MG, Santana FF, Melo LFS, Oliveira SEM, Lopes JB (2013) Revitalização da microbacia hidrográfica do Riacho Sucuruiú, em Gilbués, Piauí, Brasil. Zonas Áridas, 15 (2): 272289. DOI: http://dx.doi.org/10.21704/za.v15i2.119

Santos JHDS, Ferreira RLC, Silva JAAD, Souza ALD, Santos EDS, Meunier IMJ (2004) Distinção de grupos ecológicos de espécies florestais por meio de técnicas multivariadas. Revista Árvore 28 (3): 387396. DOI: https://doi.org/10.1590/S0100$\underline{67622004000300010}$

Santos MCVD, Silva NMD, Rodrigues R (2015) Análise comparativa da recuperação de trecho de mata ciliar, Rio Cuiabá, Cuiabá-MT em quadrantes submetidos a diferentes condições de manejo. UNIVAG, 2: 102-113.
Schievenin DF, Tonello KC, Silva DA, Valente ROA, Faria LC, Thiersch CR (2012) Monitoramento de indicadores de uma área de restauração florestal em Sorocaba-SP. Científica Eletrônica de Engenharia Florestal, 1 (19): 95-108.

Sergipe (2015) Centro de Meteorologia da Secretaria de Estado do Meio Ambiente e dos Recursos Hídricos - Superintendência de Recursos Hídricos, 2015. Estação: Boquim-SE. Disponível em:http://sinda.crn2.inpe.br/PCD/SITE/novo/site/hi storico/passo2.php Acesso: julho de 2015.

Silva ICD, Oliveira RMD, Silva TFD (2012) Evidências da degradação ambiental na mata ciliar do rio Itapororoca, no município de Itapororoca-PB, Revista Geonorte, Edição Especial, 1 (4): 663-675.

Silva KD, Martins SV, Miranda Neto A, Demolinari RDA, Lopes AT (2016) Restauração florestal de uma Mina de Bauxita: avaliação do desenvolvimento das espécies arbóreas plantadas. Floresta $e$ Ambiente, 4: 1-11. DOI: http://dx.doi.org/10.1590/2179-8087.142515

Silva NSDL, Silva NOD, Schliewe MA, Darosci AAB (2015) Desenvolvimento de plântulas em área degradada de mata de galeria no Parque Municipal Mata da Bica, Formosa-GO. RETEC, 8 (1): 17-35.

Varjabedian R (2010) Lei da Mata Atlântica: retrocesso ambiental. Estudos Avançados, 24 (68): 147-160. DOI: https://doi.org/10.1590/S010340142010000100013 